DESY-97-205

October 1997

\title{
QCD Corrections to Double and Single Spin Asymmetries in Vector Boson Production at Polarized Hadron Colliders
}

\author{
T. Gehrmann \\ DESY, Theory Group, D-22603 Hamburg, Germany
}

\begin{abstract}
The production of $W^{ \pm}$and $Z^{0}$ bosons in collisions of longitudinally polarized protons allows to access various combinations of the polarized quark distributions from the study of double and single spin asymmetries. We compute the $\mathcal{O}\left(\alpha_{s}\right)$ corrections to these asymmetries as function of the vector boson rapidity $y$ in the hadron-hadron centre-of-mass frame. Detailed studies of the numerical impact of the next-to-leading order corrections are presented. The theoretical uncertainties of an extraction of polarized quark distributions from future vector boson production data are investigated in detail.
\end{abstract}

PACS: 13.88.+e;13.75.Cs;13.85.Qk;12.38.Bx

Keywords: Vector boson production, polarization, hadron-hadron collisions. 


\section{Introduction}

The experimental knowledge on the spin structure of the nucleon has up to now been largely restricted to measurements [1] of the polarized structure function $g_{1}^{p, n, d}\left(x, Q^{2}\right)$. This structure function probes a particular charge-weighted combination of the polarized quark distributions. Its mere knowledge is therefore insufficient to disentangle the contributions of valence and sea quarks to the nucleon spin and for a further decomposition of the light quark sea into different flavours. A recent study of asymmetries in semi-inclusive hadron production by the SMC collaboration [2] yielded some additional constraints on the valence and sea quark polarization. These data are however still insufficient for a simultaneous determination of all different polarized quark and antiquark distributions.

One of the major future projects in the study of the nucleon spin structure is the operation of the Relativistic Heavy Ion Collider (RHIC) at BNL with two colliding polarized proton beams at centre-of-mass energies of $\sqrt{S}=200 \ldots 500 \mathrm{GeV}$ [3]. The RHIC spin programme is expected to start operation three years from now and will offer new information on the polarized parton distributions from a variety of observables accessible in hadron-hadron collisions [4]. A recent overview of the physics prospects of the RHIC spin programme has been presented in [5], where estimates of the expected asymmetries and anticipated errors for the most important processes can be found. At RHIC it will be possible to access the polarized quark distributions from the study of double and single spin asymmetries in the production of $V=W^{ \pm}, Z^{0}$ vector bosons.

Up to very recently, these asymmetries have only been studied [4, 5, 6, 6] at lowest order in perturbation theory, although it is well known from the unpolarized case that perturbative corrections to vector boson production cross sections at hadron colliders are sizable [8, 9, 10, 11, 12, 13, 14]. The calculation of the next-to-leading order $\left(\mathcal{O}\left(\alpha_{s}\right)\right)$ corrections to single and double spin asymmetries as function of the vector boson rapidity $y$ in the hadron-hadron centre-of-mass frame and the study of their numerical impact is the aim of the present paper. The perturbative corrections to double spin asymmetries in vector boson production are identical to the corrections in the longitudinally polarized Drell-Yan process and can therefore be easily obtained from [15, 16]. Corrections to the corresponding single spin asymmetries have been first considered in [17] in the context of a soft gluon resummation. We shall present a rederivation of these corrections, which follows closely our earlier calculation of the QCD corrections to the double spin asymmetry in the Drell-Yan process [16].

The QCD corrections to the total asymmetries (integrated over the vector boson rapidity $y$ ) in Drell-Yan process and vector boson production at RHIC have recently been studied in [18, 19]. Comparison with these provides a strong cross-check of our results.

A fully consistent numerical study of spin asymmetries in vector boson production at next-to-leading order was until now not possible, as the polarized parton distributions were only determined at leading accuracy. With the recently calculated polarized two-loop splitting functions [20], the polarized distributions can now be determined to next-to-leading order from fits [21, 22, 23, 24] to polarized structure function data. Having a complete calculation of the two-loop splitting functions available, it is now furthermore possible to define consistent scheme transformation prescriptions [20, 23] for parton dis- 
tributions, splitting functions and parton level cross sections at next-to-leading order. Possible choices of the factorization scheme in polarized and unpolarized Drell-Yan process are elaborated in great detail in [18, 19]. In our study, we shall only work in the $\overline{M S}$ scheme (with an appropriate correction for spurious terms generated due to the chosen representation of $\gamma_{5}$ ), which is identical to the scheme used in the recent determinations of polarized parton distributions at next-to-leading order [21, 22].

In the context of the present study, we shall only consider the rapidity distributions of the produced vector bosons, not of their decay products. In the case of $Z^{0}$ boson production, identified from the dilepton decay mode, a reconstruction of the boson rapidity is indeed possible, such that a direct comparison of our results with experimental data is feasible. $W^{ \pm}$bosons can on the other hand only be detected from their $l \nu_{l}$ decay mode, where just the lepton is observed, thus rendering a direct reconstruction of the $W^{ \pm}$rapidity unfeasible. The calculation presented here for the $W^{ \pm}$bosons is therefore only a first step towards a prediction of the lepton plus missing transverse momentum distribution in polarized hadronic collisions at next-to-leading order.

This paper is structured as follows: in section 2, we define the single and double spin asymmetries accessible in vector boson production at RHIC and review briefly their phenomenology. Section 3 contains the calculation of the QCD corrections to these asymmetries. As this calculation is technically very similar to the calculation of the corrections to the polarized Drell-Yan process presented in our earlier work [16], we will only point out the differences to the Drell-Yan calculation without presenting all details of our derivation. The numerical magnitude of the corrections will be studied in detail in section 4 . Finally, section 5 contains a summary of our results and concluding remarks.

\section{Spin Asymmetries in Vector Boson Production}

The production of vector bosons in hadronic collisions can be described accurately in the narrow-width approximation (e.g. [25]) as production of an on-shell massive vector boson by quark-antiquark annihilation. Like in the case of the polarized Drell-Yan process, one can study the double spin asymmetry as a function of the rapidity of the vector boson in the hadronic centre-of-mass frame:

$$
A_{L L}(y) \equiv \frac{\mathrm{d} \Delta \sigma_{L L}}{\mathrm{~d} y} / \frac{\mathrm{d} \sigma}{\mathrm{d} y}
$$

where

$$
\begin{aligned}
\mathrm{d} \Delta \sigma_{L L} & =\frac{1}{4}\left(\mathrm{~d} \sigma^{++}-\mathrm{d} \sigma^{+-}-\mathrm{d} \sigma^{-+}+\mathrm{d} \sigma^{--}\right) \\
\mathrm{d} \sigma & =\frac{1}{4}\left(\mathrm{~d} \sigma^{++}+\mathrm{d} \sigma^{+-}+\mathrm{d} \sigma^{-+}+\mathrm{d} \sigma^{--}\right),
\end{aligned}
$$

with $(+)$ and $(-)$ denoting positive and negative hadron helicities.

The unpolarized cross section in the denominator of this asymmetry can be predicted using the known unpolarized parton distribution functions. It is evaluated at next-toleading order for $p p$ collisions at $\sqrt{S}=500 \mathrm{GeV}$ in Fig. 1, using the unpolarized parton 

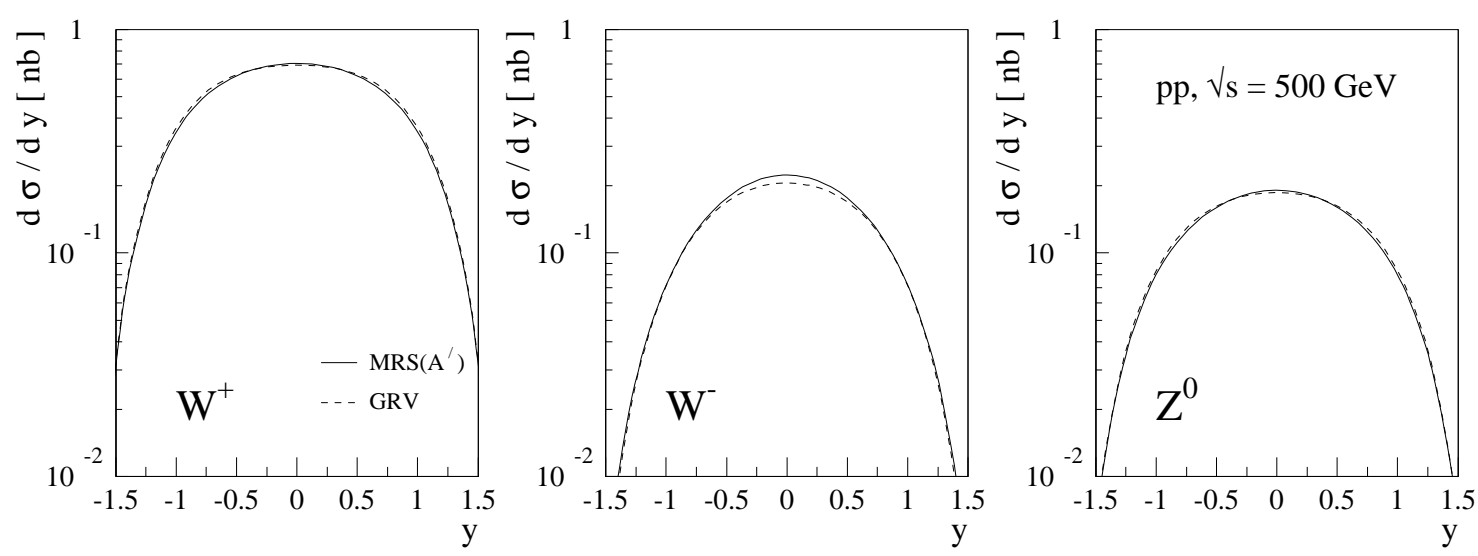

Figure 1: Unpolarized vector boson production cross sections in proton-proton collisions at $\mathrm{RHIC}(\sqrt{S}=500 \mathrm{GeV})$.

distributions from MRS(set $\mathrm{A}^{\prime}$ ) [26] and GRV [27]. The rather small discrepancy between both predictions seems to suggest that the unpolarized cross section can be predicted reliably. This is however not true in reality, since the ratio of $W^{+}$and $W^{-}$production cross sections in proton-proton collisions is proportional to the ratio of the unpolarized light antiquark distributions $\bar{d}\left(x, Q^{2}\right) / \bar{u}\left(x, Q^{2}\right)$, which is only poorly known at present. In principle, a measurement of the unpolarized $W$ boson production cross sections at RHIC could help to determine this ratio, as suggested in [7, 28]. However, by the time RHIC comes into operation, this ratio should already be well constrained from measurements of the Drell-Yan lepton pair production in proton-proton and proton-deuterium collisions [29, 30], thus enabling a reliable prediction of the unpolarized cross sections.

The phenomenology of the double spin asymmetries is best understood at leading order (and neglecting the CKM mixing factors $\left|V_{i j}\right|$ for $W^{ \pm}$production), where they can be expressed [6, 7] as ratios of polarized and unpolarized quark distributions

$$
\begin{aligned}
& A_{L L}^{W^{+}}(y)=-\frac{\Delta u\left(x_{1}^{0}, M_{W}^{2}\right) \Delta \bar{d}\left(x_{2}^{0}, M_{W}^{2}\right)+\Delta \bar{d}\left(x_{1}^{0}, M_{W}^{2}\right) \Delta u\left(x_{2}^{0}, M_{W}^{2}\right)}{u\left(x_{1}^{0}, M_{W}^{2}\right) \bar{d}\left(x_{2}^{0}, M_{W}^{2}\right)+\bar{d}\left(x_{1}^{0}, M_{W}^{2}\right) u\left(x_{2}^{0}, M_{W}^{2}\right)}, \\
& A_{L L}^{W^{-}}(y)=-\frac{\Delta d\left(x_{1}^{0}, M_{W}^{2}\right) \Delta \bar{u}\left(x_{2}^{0}, M_{W}^{2}\right)+\Delta \bar{u}\left(x_{1}^{0}, M_{W}^{2}\right) \Delta d\left(x_{2}^{0}, M_{W}^{2}\right)}{d\left(x_{1}^{0}, M_{W}^{2}\right) \bar{u}\left(x_{2}^{0}, M_{W}^{2}\right)+\bar{u}\left(x_{1}^{0}, M_{W}^{2}\right) d\left(x_{2}^{0}, M_{W}^{2}\right)}, \\
& A_{L L}^{Z^{0}}(y)=-\frac{\sum_{q}\left(v_{q}^{2}+a_{q}^{2}\right)\left[\Delta q\left(x_{1}^{0}, M_{Z}^{2}\right) \Delta \bar{q}\left(x_{2}^{0}, M_{Z}^{2}\right)+\Delta \bar{q}\left(x_{1}^{0}, M_{Z}^{2}\right) \Delta q\left(x_{2}^{0}, M_{Z}^{2}\right)\right]}{\sum_{q}\left(v_{q}^{2}+a_{q}^{2}\right)\left[q\left(x_{1}^{0}, M_{Z}^{2}\right) \bar{q}\left(x_{2}^{0}, M_{Z}^{2}\right)+\bar{q}\left(x_{1}^{0}, M_{Z}^{2}\right) q\left(x_{2}^{0}, M_{Z}^{2}\right)\right]},
\end{aligned}
$$

the distribtions being probed at

$$
x_{1,2}^{0}=\sqrt{M_{V}^{2} / S} e^{ \pm y} .
$$

At RHIC with $\sqrt{S}=500 \mathrm{GeV}$, these asymmetries are thus sensitive on $x_{1,2} \gtrsim 0.05$, where one expects the polarized quark distributions $\Delta u\left(x, Q^{2}\right)$ and $\Delta d\left(x, Q^{2}\right)$ to be dominated by contributions from valence quarks, which are reasonably well constrained from 
inclusive and semi-inclusive asymmetries in lepton-hadron scattering. The above double spin asymmetries can therefore be used as a direct probe of the polarized antiquark distributions. Even the flavour structure of the polarized quark sea can be revealed, since $W^{+}$production probes only $\Delta \bar{d}$, while $W^{-}$production is particularly sensitive on $\Delta \bar{u}$.

In addition to the above double spin asymmetries, it is also possible to define nonzero single spin asymmetries, which are induced by the parity violating couplings of the vector bosons. These asymmetries are absent in the (parity conserving) classical DrellYan process and their measurement requires only one of the incoming hadrons to be polarized. Taking hadron 1 (moving in the $+y$ direction) to be polarized and hadron 2 to be unpolarized, one can define the following single spin asymmetry

$$
A_{L}(y) \equiv \frac{\mathrm{d} \Delta \sigma_{L}}{\mathrm{~d} y} / \frac{\mathrm{d} \sigma}{\mathrm{d} y}
$$

where

$$
\mathrm{d} \Delta \sigma_{L}=\frac{1}{4}\left(\mathrm{~d} \sigma^{++}+\mathrm{d} \sigma^{+-}-\mathrm{d} \sigma^{-+}-\mathrm{d} \sigma^{--}\right)
$$

and $\mathrm{d} \sigma$ as in eq.(3) above.

At leading order (again neglecting the CKM mixing factors for $W^{ \pm}$production), one finds the following simple expressions for these single spin asymmetries [6, 7]:

$$
\begin{aligned}
A_{L}^{W^{+}}(y) & =\frac{-\Delta u\left(x_{1}^{0}, M_{W}^{2}\right) \bar{d}\left(x_{2}^{0}, M_{W}^{2}\right)+\Delta \bar{d}\left(x_{1}^{0}, M_{W}^{2}\right) u\left(x_{2}^{0}, M_{W}^{2}\right)}{u\left(x_{1}^{0}, M_{W}^{2}\right) \bar{d}\left(x_{2}^{0}, M_{W}^{2}\right)+\bar{d}\left(x_{1}^{0}, M_{W}^{2}\right) u\left(x_{2}^{0}, M_{W}^{2}\right)}, \\
A_{L}^{W^{-}}(y) & =\frac{-\Delta d\left(x_{1}^{0}, M_{W}^{2}\right) \bar{u}\left(x_{2}^{0}, M_{W}^{2}\right)+\Delta \bar{u}\left(x_{1}^{0}, M_{W}^{2}\right) d\left(x_{2}^{0}, M_{W}^{2}\right)}{d\left(x_{1}^{0}, M_{W}^{2}\right) \bar{u}\left(x_{2}^{0}, M_{W}^{2}\right)+\bar{u}\left(x_{1}^{0}, M_{W}^{2}\right) d\left(x_{2}^{0}, M_{W}^{2}\right)}, \\
A_{L}^{Z^{0}}(y) & =\frac{\sum_{q}\left(v_{q}^{2}+a_{q}^{2}\right)\left[-\Delta q\left(x_{1}^{0}, M_{Z}^{2}\right) \bar{q}\left(x_{2}^{0}, M_{Z}^{2}\right)+\Delta \bar{q}\left(x_{1}^{0}, M_{Z}^{2}\right) q\left(x_{2}^{0}, M_{Z}^{2}\right)\right]}{\sum_{q}\left(v_{q}^{2}+a_{q}^{2}\right)\left[q\left(x_{1}^{0}, M_{Z}^{2}\right) \bar{q}\left(x_{2}^{0}, M_{Z}^{2}\right)+\bar{q}\left(x_{1}^{0}, M_{Z}^{2}\right) q\left(x_{2}^{0}, M_{Z}^{2}\right)\right]} .
\end{aligned}
$$

At large and positive rapidity $y,\left(x_{1}^{0}>x_{2}^{0}\right)$, the above expressions are dominated by the first term in the numerator, since $\Delta q\left(x_{1}^{0}, Q^{2}\right) \gg \Delta \bar{q}\left(x_{1}^{0}, Q^{2}\right)$ for large $x_{1}^{0}$. The second term in the numerator is dominant for large and negative $y,\left(x_{1}^{0}<x_{2}^{0}\right)$, since $q\left(x_{2}^{0}, Q^{2}\right) \gg \bar{q}\left(x_{2}^{0}, Q^{2}\right)$ for large $x_{2}^{0}$. In practice, this means that a measurement of these single spin asymmetries at RHIC with $\sqrt{S}=500 \mathrm{GeV}$ can probe [5, 7] the polarized quark distributions for $x \gtrsim 0.2$ from $A_{L}(y>0)$ and the polarized antiquark distributions for $x \lesssim 0.12$ from $A_{L}(y<0)$. A discrimination of different quark flavours is again possible from a study of $W^{+}$and $W^{-}$boson production.

Using the double and single spin asymmetries $A_{L L}(y), A_{L}(y), A_{L}(-y)$ together with the unpolarized cross section $\mathrm{d} \sigma(y) / \mathrm{d} y$, one can calculate the cross section $\mathrm{d} \sigma^{\lambda_{1} \lambda_{2}}(y) / \mathrm{d} y$ for any combination $\lambda_{1} \lambda_{2}$ of hadron helicities. It is therefore possible to express the parity violating double spin asymmetries $A_{L L}^{\mathrm{PV}}(y)$ and $\bar{A}_{L L}^{\mathrm{PV}}(y)$ discussed in [5] by linear combinations of the asymmetries defined above. 


\section{Perturbative corrections to the polarized vector boson production cross sections}

The derivation of the $\mathcal{O}\left(\alpha_{s}\right)$ corrections to spin asymmetries in hadronic vector boson production is in many aspects similar to the calculation of the $\mathcal{O}\left(\alpha_{s}\right)$ corrections to the $y$ distribution of lepton pairs produced via the Drell-Yan process in collisions of longitudinally polarized hadrons. We have recently presented a complete derivation [16] of these corrections to the longitudinally polarized Drell-Yan process, which was following closely the unpolarized calculation of Altarelli, Ellis and Martinelli [10.

The only lowest order contribution to the Drell-Yan process comes from the annihilation of a quark-antiquark pair into a virtual photon $\gamma^{*}$ (or on-shell vector boson $V$ ) of invariant mass $M^{2}$. At next-to-leading order, one has two different contributions: the $\mathcal{O}\left(\alpha_{s}\right)$ correction (emission of a real or virtual gluon) to the $q \bar{q}$ annihilation process and the quark-gluon compton scattering process $q g \rightarrow q \gamma^{*}(V)$, where $q$ can be a quark or antiquark. Both next-to-leading order parton level cross sections contain divergences associated with collinear singularities in the initial state. These divergences can be made explicit in dimensional regularization with $d=4-2 \epsilon$, they are process independent and can be factorized into the bare parton distribution functions. In the case of the Drell-Yan process at $\mathcal{O}\left(\alpha_{s}\right)$, all infinities can be absorbed into the bare quark distribution.

The use to dimensional regularization to compute spin dependent quantities faces the problem of representing $\gamma_{5}$ in $n \neq 4$ dimensions. We use the $\gamma_{5}$-prescription [32] of 't Hooft, Veltman, Breitenlohner and Maison (HVBM), which has been consistently used in the derivation of the next-to-leading order corrections to the polarized splitting functions [20]. In this prescription, one restricts the anticommutation property of $\gamma_{5}$ to the physical four dimensions, while $\gamma_{5}$ commutes in the remaining $n-4$ dimensions. The major drawback of this formalism in the $\overline{\mathrm{MS}}$-scheme is the non-conservation of the flavour nonsinglet axial vector currents [33] due to a non-vanishing first moment of the corresponding non-singlet NLO splitting function $\Delta P_{q q,+}$. To restore the conservation of this non-singlet axial vector current, a further scheme transformation [33] of the results obtained in the HVBM formalism is needed [20]. An explicit formula for this scheme transformation, represented by a finite counterterm in the bare polarized quark distribution is given for example in 16.

Inclusive vector boson production in unpolarized hadron collisions is up to $\mathcal{O}\left(\alpha_{s}\right)$ in perturbation theory identical to the Drell-Yan process, differences due to the coupling of the $Z^{0}$ boson to a closed quark loop [9] occur only at $\mathcal{O}\left(\alpha_{s}^{2}\right)$. It should therefore be expected that the $\mathcal{O}\left(\alpha_{s}\right)$ corrections to the double polarized cross section $\mathrm{d} \Delta \sigma_{L L}(y) / \mathrm{d} y$ are identical to the corrections to the $y$ distribution of lepton pairs produced in the polarized Drell-Yan process [15, 16]. This identity could only be spoilt if the vector and axial-vector components of the vector boson couplings $\left(v_{q}\right.$ and $\left.a_{q}\right)$ to polarized quarks would acquire different higher order corrections due to the breaking of the anticommutation property of $\gamma_{5}$ in $n \neq 4$ dimensions.

Using the HVBM representation of $\gamma_{5}$, one can easily show that it is still possible to factor out the combination $v_{q}^{2}+a_{q}^{2}$ from the squared next-to-leading order matrix elements. 
The resulting matrix elements then coincide with the matrix elements appearing in the polarized Drell-Yan process up to terms which yield only contributions of $\mathcal{O}(\epsilon)$ to the final result. The $\mathcal{O}\left(\alpha_{s}\right)$ corrections to the double polarized cross section (2) are therefore indeed identical to the corrections to the polarized Drell-Yan process, as naïvely expected.

The single polarized cross section defined in $(\overline{0})$ vanishes due to parity conservation in the 'classical' Drell-Yan process, which is mediated by a virtual photon. Only the parity violating couplings of the $W^{ \pm}$and $Z^{0}$ bosons to quarks induce non-zero single spin asymmetries. In the lowest order annihilation process, one has to distinguish between the annihilation of a quark from the polarized hadron with an antiquark from the unpolarized hadron $(\Delta q+\bar{q})$ and the annihilation of an antiquark from the polarized hadron with a quark from the unpolarized hadron $(\Delta \bar{q}+q)$. The $\epsilon$-dependent parts of these lowest order cross sections, relevant for the finite terms generated in the mass factorization, are non-identical if the HVBM prescription for $\gamma_{5}$ is used:

$$
\Delta \hat{\sigma}_{L}(\Delta q+\bar{q} \rightarrow V) \sim-(1-\epsilon), \quad \Delta \hat{\sigma}_{L}(\Delta \bar{q}+q \rightarrow V) \sim(1+\epsilon)
$$

These normalization factors of the lowest order cross sections determine the terms that have to be factored out from all matrix elements of the $\mathcal{O}\left(\alpha_{s}\right)$ subprocess contributions. The factor $-(1-\epsilon)$ is required for the $(\Delta q+\bar{q})$ annihilation process and for the $(\Delta G+\bar{q})$ and $(\Delta q+G)$ gluon Compton scattering processes, while $(1+\epsilon)$ must be factored out from the $(\Delta \bar{q}+q)$ annihilation process and the $(\Delta G+q)$ and $(\Delta \bar{q}+G)$ gluon Compton scattering processes. Once the correct normalization has been factored out, the derivation of the partonic coefficient functions is identical to the derivation in the double polarized Drell-Yan process [16]. We shall only quote the final results below.

The normalization factor common to all polarized and unpolarized cross sections is

$$
N=\frac{\pi G_{F} M_{V}^{2} \sqrt{2}}{3 S}
$$

and the coupling factors read

$$
\begin{array}{cl}
c_{i j}=\left|V_{i j}\right| & \text { for } W^{ \pm}, \\
c_{i j}=\left(v_{i}^{2}+a_{i}^{2}\right) \delta_{i j} & \text { for } Z^{0} \text { unpolarized and double polarized } \\
c_{i j}=2 v_{i} a_{i} \delta_{i j} & \text { for } Z^{0} \text { single polarized } .
\end{array}
$$

The unpolarized and polarized vector boson production cross sections at next-to-leading order can be expressed in a compact analytic form as convolution of parton distributions with partonic coefficient functions:

$$
\begin{aligned}
& \frac{\mathrm{d} \sigma}{\mathrm{d} y}=N \sum_{i, j} c_{i j} \int_{x_{1}^{0}}^{1} \mathrm{~d} x_{1} \int_{x_{2}^{0}}^{1} \mathrm{~d} x_{2} \\
& \times\left\{\left[D_{q \bar{q}}^{(0)}\left(x_{1}, x_{2}, x_{1}^{0}, x_{2}^{0}\right)+\frac{\alpha_{s}}{2 \pi} D_{q \bar{q}}^{(1)}\left(x_{1}, x_{2}, x_{1}^{0}, x_{2}^{0}, \frac{M^{2}}{\mu_{F}^{2}}\right)\right]\right. \\
& \times\left\{q_{i}\left(x_{1}, \mu_{F}^{2}\right) \bar{q}_{j}\left(x_{2}, \mu_{F}^{2}\right)+\bar{q}_{i}\left(x_{1}, \mu_{F}^{2}\right) q_{j}\left(x_{2}, \mu_{F}^{2}\right)\right\}
\end{aligned}
$$




$$
\begin{aligned}
& +\frac{\alpha_{s}}{2 \pi} D_{g q}^{(1)}\left(x_{1}, x_{2}, x_{1}^{0}, x_{2}^{0}, \frac{M^{2}}{\mu_{F}^{2}}\right) G\left(x_{1}, \mu_{F}^{2}\right)\left\{q_{j}\left(x_{2}, \mu_{F}^{2}\right)+\bar{q}_{j}\left(x_{2}, \mu_{F}^{2}\right)\right\} \\
& \left.+\frac{\alpha_{s}}{2 \pi} D_{q g}^{(1)}\left(x_{1}, x_{2}, x_{1}^{0}, x_{2}^{0}, \frac{M^{2}}{\mu_{F}^{2}}\right)\left\{q_{i}\left(x_{1}, \mu_{F}^{2}\right)+\bar{q}_{i}\left(x_{1}, \mu_{F}^{2}\right)\right\} G\left(x_{2}, \mu_{F}^{2}\right)\right\}, \\
& \frac{\mathrm{d} \Delta \sigma_{L L}}{\mathrm{~d} y}=-N \sum_{i, j} c_{i j} \int_{x_{1}^{0}}^{1} \mathrm{~d} x_{1} \int_{x_{2}^{0}}^{1} \mathrm{~d} x_{2} \\
& \times\left\{\left[D_{q \bar{q}}^{(0)}\left(x_{1}, x_{2}, x_{1}^{0}, x_{2}^{0}\right)+\frac{\alpha_{s}}{2 \pi} D_{q \bar{q}}^{(1)}\left(x_{1}, x_{2}, x_{1}^{0}, x_{2}^{0}, \frac{M^{2}}{\mu_{F}^{2}}\right)\right]\right. \\
& \times\left\{\Delta q_{i}\left(x_{1}, \mu_{F}^{2}\right) \Delta \bar{q}_{j}\left(x_{2}, \mu_{F}^{2}\right)+\Delta \bar{q}_{i}\left(x_{1}, \mu_{F}^{2}\right) \Delta q_{j}\left(x_{2}, \mu_{F}^{2}\right)\right\} \\
& +\frac{\alpha_{s}}{2 \pi} \Delta D_{g q}^{(1)}\left(x_{1}, x_{2}, x_{1}^{0}, x_{2}^{0}, \frac{M^{2}}{\mu_{F}^{2}}\right) \Delta G\left(x_{1}, \mu_{F}^{2}\right)\left\{\Delta q_{j}\left(x_{2}, \mu_{F}^{2}\right)+\Delta \bar{q}_{j}\left(x_{2}, \mu_{F}^{2}\right)\right\} \\
& \left.+\frac{\alpha_{s}}{2 \pi} \Delta D_{q g}^{(1)}\left(x_{1}, x_{2}, x_{1}^{0}, x_{2}^{0}, \frac{M^{2}}{\mu_{F}^{2}}\right)\left\{\Delta q_{i}\left(x_{1}, \mu_{F}^{2}\right)+\Delta \bar{q}_{i}\left(x_{1}, \mu_{F}^{2}\right)\right\} \Delta G\left(x_{2}, \mu_{F}^{2}\right)\right\}, \\
& \frac{\mathrm{d} \Delta \sigma_{L}}{\mathrm{~d} y}=N \sum_{i, j} c_{i j} \int_{x_{1}^{0}}^{1} \mathrm{~d} x_{1} \int_{x_{2}^{0}}^{1} \mathrm{~d} x_{2} \\
& \times\left\{\left[D_{q \bar{q}}^{(0)}\left(x_{1}, x_{2}, x_{1}^{0}, x_{2}^{0}\right)+\frac{\alpha_{s}}{2 \pi} D_{q \bar{q}}^{(1)}\left(x_{1}, x_{2}, x_{1}^{0}, x_{2}^{0}, \frac{M^{2}}{\mu_{F}^{2}}\right)\right]\right. \\
& \times\left\{-\Delta q_{i}\left(x_{1}, \mu_{F}^{2}\right) \bar{q}_{j}\left(x_{2}, \mu_{F}^{2}\right)+\Delta \bar{q}_{i}\left(x_{1}, \mu_{F}^{2}\right) q_{j}\left(x_{2}, \mu_{F}^{2}\right)\right\} \\
& +\frac{\alpha_{s}}{2 \pi} \Delta D_{g q}^{(1)}\left(x_{1}, x_{2}, x_{1}^{0}, x_{2}^{0}, \frac{M^{2}}{\mu_{F}^{2}}\right) \Delta G\left(x_{1}, \mu_{F}^{2}\right)\left\{q_{j}\left(x_{2}, \mu_{F}^{2}\right)-\bar{q}_{j}\left(x_{2}, \mu_{F}^{2}\right)\right\} \\
& \left.+\frac{\alpha_{s}}{2 \pi} D_{q g}^{(1)}\left(x_{1}, x_{2}, x_{1}^{0}, x_{2}^{0}, \frac{M^{2}}{\mu_{F}^{2}}\right)\left\{-\Delta q_{i}\left(x_{1}, \mu_{F}^{2}\right)+\Delta \bar{q}_{i}\left(x_{1}, \mu_{F}^{2}\right)\right\} G\left(x_{2}, \mu_{F}^{2}\right)\right\} .
\end{aligned}
$$

The partonic coefficient functions in the above expressions are identical to the coefficient functions occurring in the $y$-distributions of the polarized and unpolarized Drell-Yan process [11, 15, 16, 31]. They read:

$$
\begin{aligned}
D_{q \bar{q}}^{(0)}\left(x_{1}, x_{2}, x_{1}^{0}, x_{2}^{0}\right)= & \delta\left(x_{1}-x_{1}^{0}\right) \delta\left(x_{2}-x_{2}^{0}\right) \\
D_{q \bar{q}}^{(1)}\left(x_{1}, x_{2}, x_{1}^{0}, x_{2}^{0}, \frac{M^{2}}{\mu_{F}^{2}}\right)= & C_{F}\left\{\delta ( x _ { 1 } - x _ { 1 } ^ { 0 } ) \delta ( x _ { 2 } - x _ { 2 } ^ { 0 } ) \left[\frac{\pi^{2}}{3}-8+2 \operatorname{Li}_{2}\left(x_{1}^{0}\right)+2 \operatorname{Li}_{2}\left(x_{2}^{0}\right)\right.\right. \\
& \left.+\ln ^{2}\left(1-x_{1}^{0}\right)+\ln ^{2}\left(1-x_{2}^{0}\right)+2 \ln \frac{x_{1}^{0}}{1-x_{1}^{0}} \ln \frac{x_{2}^{0}}{1-x_{2}^{0}}\right] \\
+ & \left(\delta\left(x_{1}-x_{1}^{0}\right)\left[\frac{1}{x_{2}}-\frac{x_{2}^{0}}{x_{2}^{2}}-\frac{x_{2}^{0} 2}{x_{2}^{2}\left(x_{2}-x_{2}^{2}\right.} x_{2}^{0}\right) \ln \frac{x_{2}^{0}}{x_{2}}\right. \\
& +\frac{x_{2}^{0} 2+x_{2}^{2}}{x_{2}^{2}}\left(\frac{\ln \left(1-x_{2}^{0} / x_{2}\right)}{x_{2}-x_{2}^{0}}\right)_{+}+\frac{x_{2}^{0} 2+x_{2}^{2}}{x_{2}^{2}} \frac{1}{\left(x_{2}-x_{2}^{0}\right)_{+}}
\end{aligned}
$$




$$
\begin{aligned}
& \left.\left.\ln \frac{2 x_{2}^{0}\left(1-x_{1}^{0}\right)}{x_{1}^{0}\left(x_{2}+x_{2}^{0}\right)}\right]+(1 \leftrightarrow 2)\right) \\
& +\frac{G^{A}\left(x_{1}, x_{2}, x_{1}^{0}, x_{2}^{0}\right)}{\left[\left(x_{1}-x_{1}^{0}\right)\left(x_{2}-x_{2}^{0}\right)\right]_{+}}+H^{A}\left(x_{1}, x_{2}, x_{1}^{0}, x_{2}^{0}\right) \\
& +\ln \frac{M^{2}}{\mu_{F}^{2}}\left\{\delta\left(x_{1}-x_{1}^{0}\right) \delta\left(x_{2}-x_{2}^{0}\right)\left[3+2 \ln \frac{1-x_{1}^{0}}{x_{1}^{0}}+2 \ln \frac{1-x_{2}^{0}}{x_{2}^{0}}\right]\right. \\
& \left.\left.+\left(\delta\left(x_{1}-x_{1}^{0}\right) \frac{x_{2}^{0}{ }^{2}+x_{2}^{2}}{x_{2}^{2}} \frac{1}{\left(x_{2}-x_{2}^{0}\right)_{+}}+(1 \leftrightarrow 2)\right)\right\}\right\} \\
& D_{g q}^{(1)}\left(x_{1}, x_{2}, x_{1}^{0}, x_{2}^{0}, \frac{M^{2}}{\mu_{F}^{2}}\right)=T_{F}\left\{\frac { \delta ( x _ { 2 } - x _ { 2 } ^ { 0 } ) } { x _ { 1 } ^ { 3 } } \left[\left(x_{1}^{02}+\left(x_{1}-x_{1}^{0}\right)^{2}\right) \ln \frac{2\left(x_{1}-x_{1}^{0}\right)\left(1-x_{2}^{0}\right)}{\left(x_{1}+x_{1}^{0}\right) x_{2}^{0}}\right.\right. \\
& \left.+2 x_{1}^{0}\left(x_{1}-x_{1}^{0}\right)\right]+\frac{G^{C}\left(x_{1}, x_{2}, x_{1}^{0}, x_{2}^{0}\right)}{\left(x_{2}-x_{2}^{0}\right)_{+}}+H^{C}\left(x_{1}, x_{2}, x_{1}^{0}, x_{2}^{0}\right) \\
& \left.+\ln \frac{M^{2}}{\mu_{F}^{2}}\left\{\frac{\delta\left(x_{2}-x_{2}^{0}\right)}{x_{1}^{3}}\left(x_{1}^{02}+\left(x_{1}-x_{1}^{0}\right)^{2}\right)\right\}\right\} \text {, } \\
& D_{q g}^{(1)}\left(x_{1}, x_{2}, x_{1}^{0}, x_{2}^{0}, \frac{M^{2}}{\mu_{F}^{2}}\right)=D_{g q}^{(1)}\left(x_{2}, x_{1}, x_{2}^{0}, x_{1}^{0}, \frac{M^{2}}{\mu_{F}^{2}}\right) \\
& \Delta D_{g q}^{(1)}\left(x_{1}, x_{2}, x_{1}^{0}, x_{2}^{0}, \frac{M^{2}}{\mu_{F}^{2}}\right)=T_{F}\left\{\frac { \delta ( x _ { 2 } - x _ { 2 } ^ { 0 } ) } { x _ { 1 } ^ { 2 } } \left[\left(2 x_{1}^{0}-x_{1}\right) \ln \frac{2\left(x_{1}-x_{1}^{0}\right)\left(1-x_{2}^{0}\right)}{\left(x_{1}+x_{1}^{0}\right) x_{2}^{0}}\right.\right. \\
& \left.+2\left(x_{1}-x_{1}^{0}\right)\right]+\frac{\Delta G^{C}\left(x_{1}, x_{2}, x_{1}^{0}, x_{2}^{0}\right)}{\left(x_{2}-x_{2}^{0}\right)_{+}}+H^{C}\left(x_{1}, x_{2}, x_{1}^{0}, x_{2}^{0}\right) \\
& \left.+\ln \frac{M^{2}}{\mu_{F}^{2}}\left\{\frac{\delta\left(x_{2}-x_{2}^{0}\right)}{x_{1}^{2}}\left(2 x_{1}^{0}-x_{1}\right)\right\}\right\} \text {, } \\
& \Delta D_{q g}^{(1)}\left(x_{1}, x_{2}, x_{1}^{0}, x_{2}^{0}, \frac{M^{2}}{\mu_{F}^{2}}\right)=\Delta D_{g q}^{(1)}\left(x_{2}, x_{1}, x_{2}^{0}, x_{1}^{0}, \frac{M^{2}}{\mu_{F}^{2}}\right) \text {, }
\end{aligned}
$$

where

$$
\begin{aligned}
G^{A}\left(x_{1}, x_{2}, x_{1}^{0}, x_{2}^{0}\right) & =\frac{2\left(x_{1} x_{2}+x_{1}^{0} x_{2}^{0}\right)\left(x_{1}^{0}{ }^{2} x_{2}^{0} 2+x_{1}^{2} x_{2}^{2}\right)}{x_{1}^{2} x_{2}^{2}\left(x_{1}+x_{1}^{0}\right)\left(x_{2}+x_{2}^{0}\right)} \\
H^{A}\left(x_{1}, x_{2}, x_{1}^{0}, x_{2}^{0}\right) & =-\frac{4 x_{1}^{0} x_{2}^{0}\left(x_{1}^{0} x_{2}^{0}+x_{1} x_{2}\right)}{x_{1} x_{2}\left(x_{1} x_{2}^{0}+x_{2} x_{1}^{0}\right)^{2}} \\
G^{C}\left(x_{1}, x_{2}, x_{1}^{0}, x_{2}^{0}\right) & =\frac{2 x_{2}^{0}\left(x_{1}^{0} x_{2}^{0} x^{2}+\left(x_{1}^{0} x_{2}^{0}-x_{1} x_{2}\right)^{2}\right)\left(x_{1}^{0} x_{2}^{0}+x_{1} x_{2}\right)}{x_{1}^{3} x_{2}^{2}\left(x_{1} x_{2}^{0}+x_{2} x_{1}^{0}\right)\left(x_{2}+x_{2}^{0}\right)} \\
H^{C}\left(x_{1}, x_{2}, x_{1}^{0}, x_{2}^{0}\right) & =\frac{2 x_{1}^{0} x_{2}^{0}\left(x_{1}^{0} x_{2}^{0}+x_{1} x_{2}\right)\left(x_{1} x_{1}^{0} x_{2}^{2}+x_{1}^{0} x_{2}^{0}\left(x_{1} x_{2}^{0}+2 x_{1}^{0} x_{2}\right)\right)}{x_{1}^{2} x_{2}^{2}\left(x_{1} x_{2}^{0}+x_{2} x_{1}^{0}\right)^{3}} \\
\Delta G^{C}\left(x_{1}, x_{2}, x_{1}^{0}, x_{2}^{0}\right) & =\frac{2 x_{2}^{0}\left(2 x_{1}^{0} x_{2}^{0}-x_{1} x_{2}\right)\left(x_{1}^{0} x_{2}^{0}+x_{1} x_{2}\right)}{x_{1}^{2} x_{2}\left(x_{1} x_{2}^{0}+x_{2} x_{1}^{0}\right)\left(x_{2}+x_{2}^{0}\right)}
\end{aligned}
$$


The occurrence of double polarized and unpolarized coefficient functions in the single polarized vector boson production cross section can be understood by simple arguments. The $W$-boson couples only to one particular helicity of the incoming quarks. The unpolarized, single and double polarized $q \bar{q}$ cross sections receive therefore only a single contribution from one particular configuration of quark and antiquark helicities and are thus identical up to an overall sign. In the case of quark-gluon Compton scattering, both different gluon helicities can contribute; the $q g$ cross sections thus receive contributions from two different helicity combinations and the unpolarized and double polarized parton cross sections are different. In the case of the single polarized cross section, it is easy to identify the polarized quark-unpolarized gluon contribution with the fully unpolarized subprocess, and the polarized gluon-unpolarized quark contribution with the fully polarized subprocess. These identifications are less obvious for the $Z^{0}$ boson production, but can be made explicit if the $Z^{0}$ couplings to the different quark handednesses are separated.

The partonic coefficient functions appearing in the single polarized vector boson cross sections have been first studied by Weber in [17], where they were obtained as a by-product in the calculation of the soft gluon resummation to these single polarized cross sections. Our results are in a simpler form than the ones given in [17] and differ both in the polarized gluon-unpolarized quark and the polarized quark-unpolarized gluon subprocesses. The discrepancy in the former coefficient can be attributed to a different factorization scheme used for the polarized gluon distribution in [17]; the discrepancy in the latter can only be understood to be due to a non-conventional normalization of the number of unpolarized gluon states in [17]. A consistency check of our results is given by the quark helicity arguments discussed above. These explain the occurrence of the unpolarized and double polarized coefficient functions in the expression for the double polarized cross section. Moreover, integration of our results over $y$ yields the QCD corrections to the inclusive asymmetries, which have been recently calculated by Kamal in [19]. Expressing the final results of [19] in the $\overline{\mathrm{MS}}_{H C^{-}}$-scheme (which is identical to our implementation of the $\overline{\mathrm{MS}}$ scheme), we find complete agreement with our results integrated over $y$.

\section{Numerical results}

The numerical impact of the next-to-leading order corrections derived in the previous section can be illustrated by evaluating the asymmetries (11) and (6) using recent next-toleading order parameterizations of polarized and unpolarized parton distributions.

When using the polarized GS parton distribution functions [21], we take $\Lambda_{n_{f}=4}^{\mathrm{QCD}}=$ $231 \mathrm{MeV}$, the corresponding unpolarized cross sections are then evaluated using the unpolarized MRS parton distribution functions set $\mathrm{A}^{\prime}$ [26]. The polarized GRSV distributions [22] are consistently used in combination with the unpolarized distributions from GRV [27] and for $\Lambda_{n_{f}=4}^{\mathrm{QCD}}=200 \mathrm{MeV}$. If not stated otherwise, we shall always use $\mu_{F}=M$; the strong coupling constant $\alpha_{s}$ is evaluated at $\mu_{F}$. All results in this section are obtained

for longitudinally polarized proton-proton collisions at RHIC $(\sqrt{S}=500 \mathrm{GeV})$; the single spin asymmetries are always obtained for the configuration where the polarized proton is moving in the $+y$ direction. 

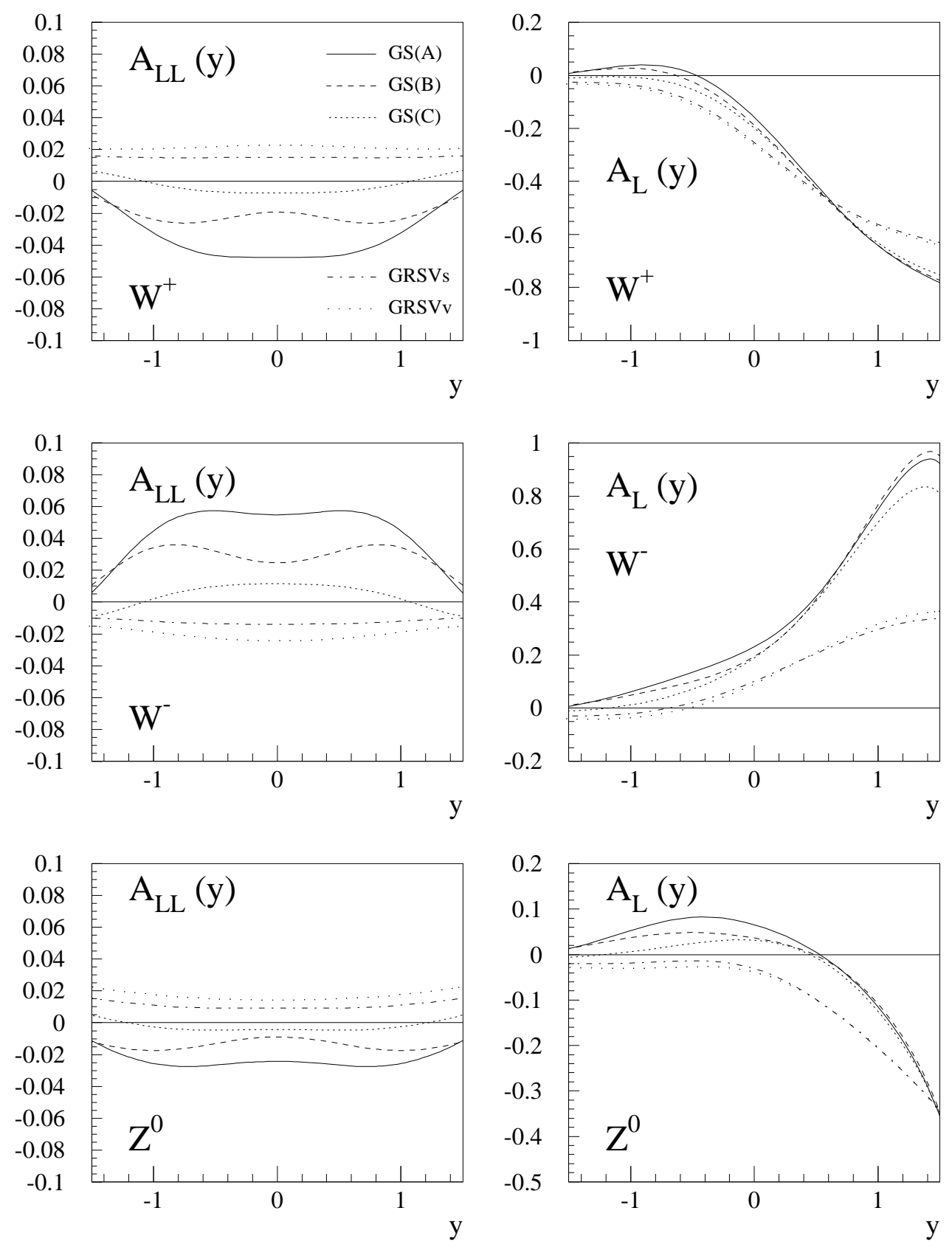

Figure 2: Predictions for double (left) and single (right) spin asymmetries in vector boson production in $p p$ collisions at $\sqrt{s}=500 \mathrm{GeV}$ obtained for different parameterizations of the next-to-leading order polarized parton densities.

It must be kept in mind that all present day parameterizations of the polarized parton distributions are fitted only to data on the structure function $g_{1}^{p, d, n}\left(x, Q^{2}\right)$. These data only provide very loose constraints on the polarized sea quark distributions at large $x$ 
and are clearly insufficient for a flavour decomposition of the polarized light quark sea. Consequently, our present knowledge on the polarized sea quark distributions is very poor. This uncertainty is illustrated in Figure 2 showing the vector boson production asymmetries $A_{L L}(y)$ and $A_{L}(y)$ evaluated at next-to-leading order using the polarized parton distribution functions of GS(A-C) 21] and GRSVs,v 22]. A more extensive study at lowest order has recently been presented in [5].

Based on these present parameterizations, it is not possible to predict sign or magnitude of the double spin asymmetry $A_{L L}(y)$ or of the single spin asymmetry $A_{L}(y<0)$; both asymmetries being dominated by contributions from the polarized sea distributions in the nucleon. From the ratio of polarized to unpolarized sea quark distributions in present parameterizations, it can only be estimated that $\left|A_{L L}(y)\right| \lesssim 0.06$ and $\left|A_{L}(y<0)\right| \lesssim 0.3$. Only $A_{L}(y>0)$ is dominated by the behaviour of the polarized valence quarks at relatively large $x$, which is reasonably well constrained by the present $g_{1}^{p, d, n}\left(x, Q^{2}\right)$ data. Consequently, the different parameterizations yield similar predictions for $A_{L}(y>0)$, a sizable discrepancy can only be seen for $W^{-}$production. This discrepancy is related to a still sizable difference in the $\Delta d_{v}\left(x, Q^{2}\right)$ distributions in the GRSV and GS parametrizations and will most likely be sorted out in the near future when new precision data on the deuteron spin structure function $g_{1}^{d}\left(x, Q^{2}\right)$ will become available.

The above discussion clearly shows that our present knowledge on the polarized quark distributions is still insufficient for precise predictions of asymmetries in vector boson production at RHIC. The following studies of the numerical effects of the next-to-leading order corrections can therefore only illustrate the qualitative effects of these corrections without making definite, quantitative predictions for asymmetries or $K$-factors.

The contributions of the individual subprocesses $(q \bar{q}-$ annihilation at leading and nextto-leading order and quark-gluon Compton scattering) to the polarized vector boson cross sections are shown in Fig. 3. All polarized subprocess cross sections are obtained with the polarized GS(A) parton distributions and are normalized to the full unpolarized cross section at next-to-leading order. In the case of the quark-gluon Compton process, we distinguish moreover the case where the gluon originates from the proton moving in $-y$ direction $(q g)$ and the case where the gluon originates from the proton moving in $+y$ direction $(g q)$. This distinction is irrelevant for $\Delta \sigma_{L L}(y)$, it becomes however important for $\Delta \sigma_{L}(y)$, where the $q g$ process probes $\Delta q\left(x_{1}, Q^{2}\right) G\left(x_{2}, Q^{2}\right)$ while the $g q$ process is sensitive on $\Delta G\left(x_{1}, Q^{2}\right) q\left(x_{2}, Q^{2}\right)$.

The numerical impact of the individual contributions to both $\Delta \sigma_{L}(y)$ and $\Delta \sigma_{L L}(y)$ is similar to the impact of these contributions in the ordinary polarized Drell-Yan process [16]. The $\mathcal{O}\left(\alpha_{s}\right)$ correction to the $q \bar{q}$-annihilation process enhances significantly the lowest order prediction while the quark-gluon Compton process contributes with a sign opposite to the annihilation process. A definite prediction of the relative magnitude of these two next-to-leading order processes is however only possible for $\Delta \sigma_{L}(y>0)$, as we shall explain below.

The quark-gluon Compton contributions to $\Delta \sigma_{L}(y)$ arise mainly from the $q g$ configuration for $y>0$ and from the $g q$ configuration for $y<0$. Consequently, an extraction of the large $x$ behaviour of the polarized quark distributions at next-to-leading order from a measurement of $A_{L}(y>0)$ will not suffer from the uncertainty on the polarized 

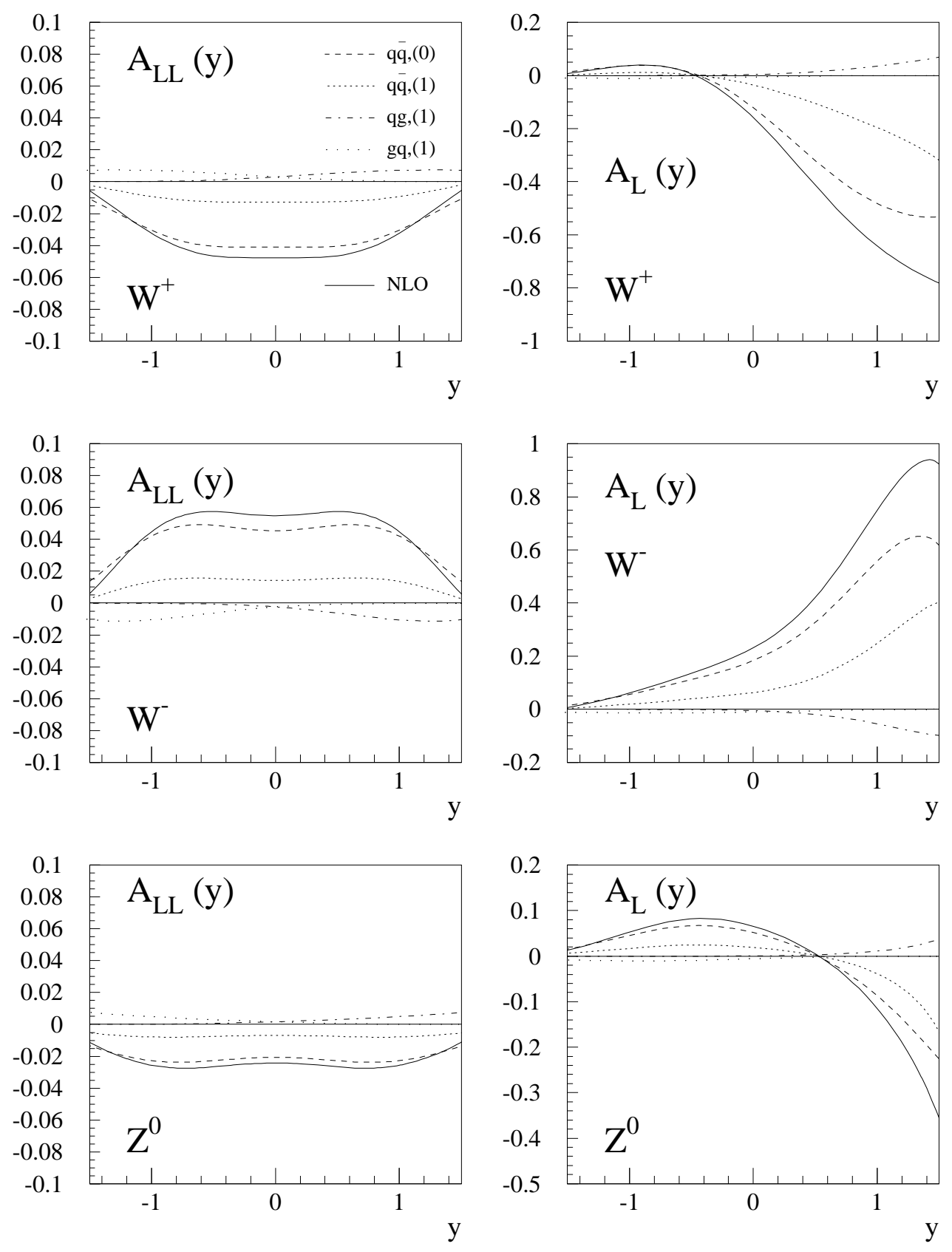

Figure 3: Contributions of the individual parton level subprocesses to the double (left) and single (right) polarized vector boson production cross sections in $p p$ collisions at $\sqrt{s}=500 \mathrm{GeV}$. All polarized cross sections are normalized to the full unpolarized cross section at next-to-leading order.

gluon distribution, since only the known unpolarized gluon distribution contributes in the $q g$ configuration. The numerical magnitude of the next-to-leading order corrections 

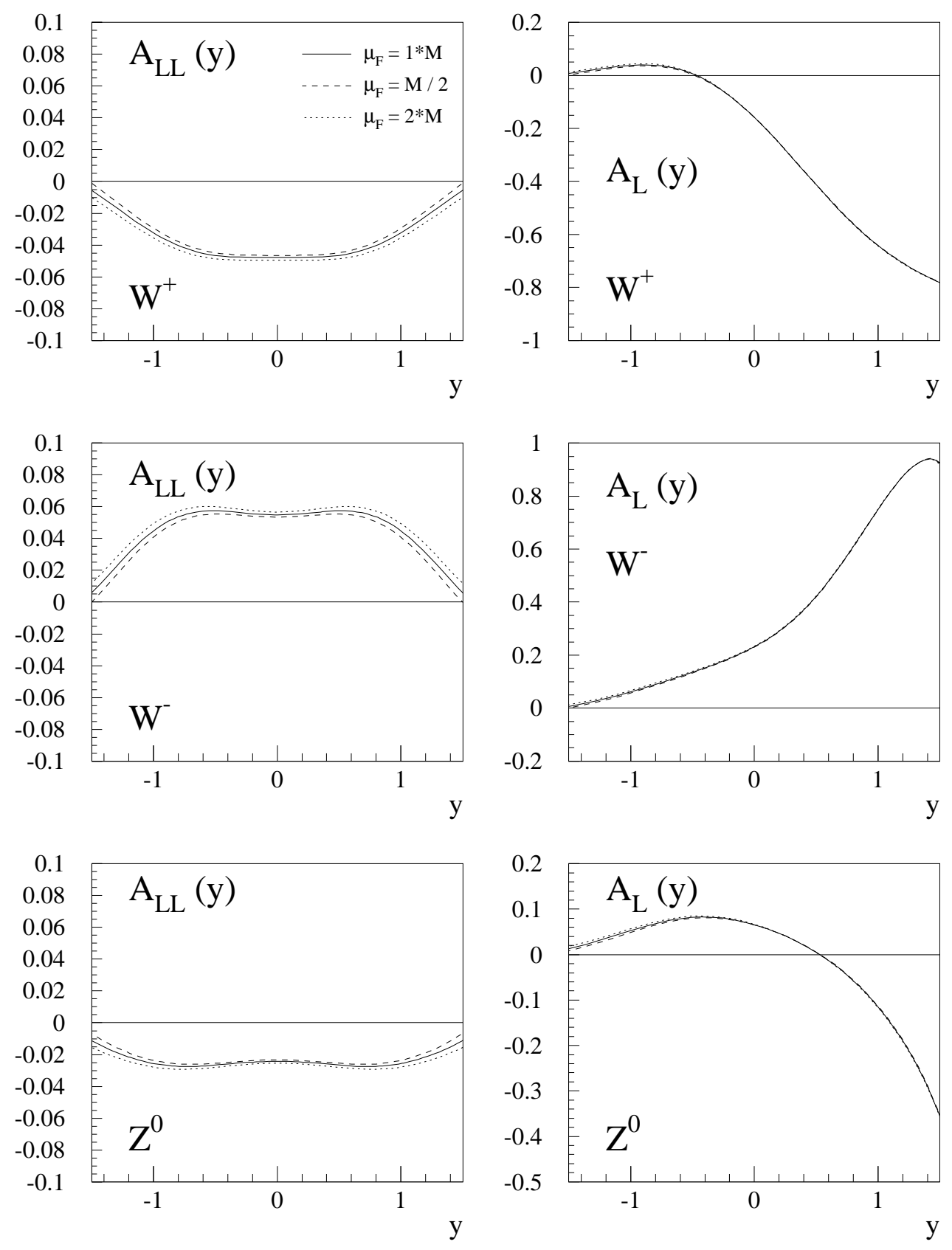

Figure 4: Variation of the double (left) and single (right) spin asymmetries in vector boson production in $p p$ collisions at $\sqrt{s}=500 \mathrm{GeV}$ under variation of the mass factorization scale.

to $A_{L}(y>0)$ can therefore predicted reliably: inclusion of these corrections enhances $\left|\Delta \sigma_{L}(y>0)\right|$ by about $15-25 \%$ compared to its lowest order value.

A quantification of the next-to-leading corrections to $\Delta \sigma_{L L}(y)$ and $\Delta \sigma_{L}(y<0)$ requires on the other hand some knowledge on the magnitude of the polarized gluon distribution 
$\Delta G\left(x, Q^{2}\right)$, which enters these cross sections in the quark-gluon Compton scattering process. The same problem was already encountered in the QCD corrections to the longitudinally polarized Drell-Yan process [16]. It illustrates that any extraction of the polarized sea quark distributions at next-to-leading order requires at least some order-of-magnitude knowledge on the polarized gluon distribution.

The change of physical quantities such as cross sections or asymmetries under variations of the (unphysical) mass factorization scale $\mu_{F}$ enables an estimate of the numerical importance of unknown higher order corrections. We quantify this uncertainty in Fig. 4 , showing the double and single spin asymmetries in vector boson production for $\mu_{F}=0.5,1,2 M_{V}$. It can be seen that the absolute value of $A_{L L}(y)$ changes by less than 0.005 in the central region, the variations become slightly larger in the region where $y$ approaches its kinematic limit, but they never exceed 0.01. The variations in $A_{L}(y>0)$ are even smaller and do not exceed 0.004, they become larger in $A_{L}(y<0)$, where they can amount up to 0.01. All these variations are however significantly smaller than the difference between predictions for $A_{L L}(y)$ and $A_{L}(y)$ obtained with various parameterizations of the polarized parton distribution functions (cf. Fig. 2). It must be emphasized that the relative smallness of these observed variations with the mass factorization scale arises partly due to the fact that the scale dependence of polarized and unpolarized cross sections largely compensate when taking their ratio in the asymmetry. The unpolarized vector boson production cross sections vary by about $15 \%$ between $\mu_{F}=0.5 M_{V}$ and $\mu_{F}=2 M_{V}$.

\section{Conclusions}

We have presented a complete calculation of the perturbative $\mathcal{O}\left(\alpha_{s}\right)$ corrections to double $\left(A_{L L}(y)\right)$ and single $\left(A_{L}(y)\right)$ spin asymmetries in massive vector boson production in collisions of longitudinally polarized hadrons. The results can be expressed in simple analytic form and are given in eqs.(12) and (13). These corrections are of particular importance for the extraction of the polarized sea quark distributions from measurements of vector boson production asymmetries at RHIC.

The next-to-leading order terms contributing to $A_{L L}(y)$ agree with the corrections to this asymmetry in the longitudinally polarized Drell-Yan process [15, 16, as naively expected.

Corrections to $A_{L}(y)$ had been first considered by Weber 17 in the context of a soft gluon resummation to single spin asymmetries. While our results for the $\Delta q+\bar{q}, \Delta \bar{q}+q$ and $\Delta G+q$ next-to-leading order subprocesses agree (after suitable change of factorization scheme) with [17], we obtain a different result for the $\Delta q+G$ subprocess (polarized quark-unpolarized gluon). This difference could be attributed to a non-conventional normalization of the gluonic polarization sum used in [17]. A check of our results is given by the fact that all single polarized subprocess cross sections can be directly related to subprocess cross sections in the double polarized or unpolarized case. This behaviour should be expected due to the helicity structure of the vector boson couplings to quarks. A further check is the integration of our results over the rapidity $y$, which reproduces the 
recent results of [19] while showing the same above discrepancy with [17].

We have demonstrated that the numerical impact of these corrections on the polarized vector boson cross sections is very similar to the impact of the next-to-leading order corrections in the unpolarized case. The corrections due to the individual subprocesses turn out to be sizable. However, a $K$-factor between leading and next-to-leading order results can at present only be predicted for $A_{L}(y>0)$, which is insensitive to the yet unknown magnitude of the polarized gluon distribution.

With the knowledge of the next-to-leading order corrections, it is furthermore possible to quantify the uncertainty of the theoretical prediction due to the choice of factorization scale and hence the theoretical error on a measurement of the polarized quark distributions. We have demonstrated that the absolute value of $A_{L L}(y)$ varies by less than 0.005 in the central region under change of the factorization scale. The variations in $A_{L}(y)$ are only sizable for $y<0$, where they amount up to 0.01. These variations are however significantly smaller than the difference between predictions obtained for $A_{L L}(y)$ and $A_{L}(y)$ using different parameterizations for the polarized parton distribution functions.

To summarize, the calculations presented in this paper allowed us to investigate two possible sources of theoretical uncertainty on an extraction of the polarized quark distributions from double and single spin asymmetries in vector boson production at RHIC. We found that the variation of the mass factorization scale had only minor effects on all asymmetries, thus indicating that the effect from unknown higher order terms can be expected to be small. The effects on these asymmetries from the yet unknown polarized gluon distribution were on the other hand found to be sizable for both $A_{L L}(y)$ and $A_{L}(y<0)$, while being negligible in $A_{L}(y>0)$. This observation illustrates that a consistent extraction of the next-to-leading order polarized sea quark distributions from vector boson production at RHIC will require at least some order-of-magnitude information on the polarized gluon distribution, which is to be obtained from other processes.

\section{Acknowledgements}

Part of the work presented in this paper has been carried out during a visit to the Fermilab Theory Group, whose kind hospitality is gratefully acknowledged. The author would moreover like to thank W. Vogelsang for clarifying discussions on Ref. [17].

\section{References}

[1] SLAC-Yale collaboration: M.J. Alguard et al., Phys. Rev. Lett. 37 (1976) 1261; G. Baum et al., Phys. Rev. Lett. 45 (1980) 2000; 51 (1983) 1135.

EMC collaboration: J. Ashman et al., Nucl. Phys. B328 (1989) 1.

SLAC-E142 collaboration: P.L. Anthony et al., Phys. Rev. Lett. 71 (1993) 959; Phys. Rev. D54 (1996) 6620.

SMC collaboration: B. Adeva et al., Phys. Lett. B302 (1993) 533; D. Adams et al., Phys. Lett. B329 (1994) 399; B357 (1995) 248; B396 (1997) 338; preprints CERNPPE-97-022 (hep-ex/9702005); CERN-PPE-97-118. 
SLAC-E143 collaboration: K. Abe et al., Phys. Rev. Lett. 74 (1995) 346; 75 (1995) 25; 78 (1997) 815; Phys. Lett. B364 (1995) 61.

HERMES collaboration: K. Ackerstaff et al., Phys. Lett. B404 (1997) 383.

SLAC-E154 collaboration: K. Abe et al., Phys. Rev. Lett. 79 (1997) 26.

[2] SMC collaboration: B. Adeva et al., Phys. Lett. B369 (1996) 93; E.M. Kabuß for the SMC collaboration, Proceedings of the 5th International Workshop on "Deep Inelastic Scattering and QCD” (DIS '97), Chicago, 1997, ed. J. Repond, AIP Conference Proceedings (New York, 1997), to appear.

[3] RHIC-SPIN collaboration: M. Beddo et al., proposal, BNL, 1992.

[4] C. Borrely, J. Soffer, F.M. Renard and P. Taxil, Phys. Rep. 177 (1989) 319.

[5] J. Soffer and J.M. Virey, Marseille Preprint CPT-97/P.3495 (hep-ph/9706229).

[6] E. Leader and K. Sridhar, Phys. Lett. B311 (1993) 324.

[7] C. Bourrely and J. Soffer, Phys. Lett. B314 (1993) 132.

[8] G. Altarelli, R.K. Ellis and G. Martinelli, Nucl. Phys. B143 (1978) 521, Erratum B146 (1978) 544.

J. Abad and B. Humpert, Phys. Lett. 80B (1979) 286.

J. Kubar-André and F.E. Paige, Phys. Rev. D19 (1979) 221.

B. Humpert and W.L. van Neerven, Phys. Lett. 84B (1979) 327, Erratum 85B (1979) 471; 89B (1979) 69; Nucl. Phys. B184 (1981) 225.

[9] A.P. Contogouris and J. Kripfganz, Phys. Rev. D20 (1979) 2295.

A.N. Schellekens and W.L. van Neerven, Phys. Rev. D21 (1980) 2619; D22 (1980) 1623.

T. Matsuura and W.L. van Neerven, Z. Phys. C38 (1988) 623.

T. Matsuura, S.C. van der Marck and W.L. van Neerven, Phys. Lett. B211 (1988) 171; Nucl. Phys. B319 (1989) 570.

R. Hamberg, T. Matsuura and W.L. van Neerven, Nucl. Phys. B345 (1990) 331; B359 (1991) 343.

W.L. van Neerven and E.B. Zijlstra, Nucl. Phys. B382 (1992) 11.

[10] G. Altarelli, R.K. Ellis and G. Martinelli, Nucl. Phys. B157 (1979) 461.

[11] J. Kubar, M. le Bellac, J.L. Meunier and G. Plaut, Nucl. Phys. B175 (1980) 251.

[12] P. Arnold and M.H. Reno, Nucl. Phys. B319 (1989) 37.

R.J. Gonsalves, J. Pawlowski and C.F. Wai, Phys. Rev. D40 (1989) 2245.

[13] E. Mirkes, J.G. Körner and G.A. Schuler, Phys. Lett. B259 (1991) 151.

[14] H. Baer and M.H. Reno, Phys. Rev. D43 (1991) 2892.

W.T. Giele, E.W.N. Glover and D.A. Kosower, Nucl. Phys. B403 (1993) 633. 
[15] A. Weber, Nucl. Phys. B382 (1992) 63.

[16] T. Gehrmann, Nucl. Phys. B498 (1997) 245.

[17] A. Weber, Nucl. Phys. B403 (1993) 545.

[18] B. Kamal, Phys. Rev. D53 (1996) 1142.

[19] B. Kamal, preprint hep-ph/9710374.

[20] R. Mertig and W.L. van Neerven, Z. Phys. C70 (1996) 637.

W. Vogelsang, Phys. Rev. D54 (1996) 2023; Nucl. Phys. B475 (1996) 47.

[21] T. Gehrmann and W.J. Stirling, Phys. Rev. D53 (1996) 6100.

[22] M. Glück, E. Reya, M. Stratmann and W. Vogelsang, Phys. Rev. D53 (1996) 4775.

[23] R.D. Ball, S. Forte and G. Ridolfi, Phys. Lett. B378 (1996) 255.

[24] G. Altarelli, R.D. Ball, S. Forte and G. Ridolfi, Nucl. Phys. B496 (1997) 337;

SLAC-E154 collaboration: K. Abe et al., Phys. Lett. B405 (1997) 180;

E. Leader, A.V. Sidorov and D.B. Stamenov, preprint hep-ph/9708335.

[25] R.K. Ellis, W.J. Stirling and B.R. Webber, QCD and Collider Physics, Cambridge University Press (Cambridge, 1996).

[26] A.D. Martin, R.G. Roberts and W.J. Stirling, Phys. Lett. B354 (1995) 155.

[27] M. Glück, E. Reya and A. Vogt, Z. Phys. C67 (1995) 433.

[28] C. Borrely and J. Soffer, Nucl. Phys. B423 (1994) 329.

[29] S.D. Ellis and W.J. Stirling, Phys. Lett. B256 (1991) 258.

[30] NA51 collaboration: A. Baldit et al., Phys. Lett. B332 (1994) 244;

R. Towell for the Fermilab-E866 collaboration, Proceedings of the 5th International Workshop on "Deep Inelastic Scattering and QCD” (DIS '97), Chicago, 1997, ed. J. Repond, AIP Conference Proceedings (New York, 1997), to appear.

[31] P. Sutton, A.D. Martin, W.J. Stirling and R.G. Roberts, Phys. Rev. D45 (1992) 2349.

[32] G. t'Hooft and M. Veltman, Nucl. Phys. B44 (1972) 189.

P. Breitenlohner and D. Maison, Comm. Math. Phys. 52 (1977) 11.

[33] S. Larin, Phys. Lett. B303 (1993) 113. 